\title{
Participación ciudadana \\ y gobierno electrónico. \\ Realidades y perspectivas
}

\section{Citizen participation and e-government. Realities and perspectives}

\section{Lucero Liliana Cabrera Benavides*}

\footnotetext{
* Socióloga y magíster en Docencia Universitaria por la Universidad de Nariño, Pasto, Colombia. Maestrante en Política Comparada, Flacso, Ecuador. Docente a tiempo completo en la Universidad Cesmag, Pasto, Colombia.

$\triangle$ luceroliliana08@hotmail.com https://orcid.org/0000-00027597-9693
}

\section{RECIBID O: 29.7.2020}

\section{Resumen}

El artículo se enfoca en la participación ciudadana que se pretende implementar mediante el portal electrónico de la Gobernación de Nariño, denominado GANA. El fin es conocer el avance de participación en los portales electrónicos de gobierno en las dimensiones informativa, interactiva, inclusiva y de control. El escrito examina el diseño de participación ciudadana de gobierno electrónico y las expectativas de los diseñadores, colaboradores y usuarios del portal electrónico, para lo cual se realizaron entrevistas semiestructuradas y grupos focales. Los resultados de investigación revelan que el diseño corresponde en general a la participación informativa, a excepción de herramientas digitales que se encuentran en el portal, como Justicia Abierta, que ofrece a la comunidad asesoría jurídica virtual de estudiantes de Derecho, y GANA Municipales, dirigida a la elección popular de proyectos, que en ocasiones brinda medios de interacción con la comunidad. Palabras claves: gobierno electrónico, participación social, sociedad civil, tecnología de la información, Colombia.

\section{Abstract}

The article focuses on citizen participation intended to be implemented through the electronic portal of the government of Nariño, called GANA. The aim is to know 
the progress of civil participation through electronic government portals considering four dimensions: information, interaction, inclusion and control. The paper examines the e-portal's design in terms of civil participation and the expectations of designers, collaborators and users. Semi-structured interviews and focus groups were conducted to achieve that aim. The results reveal that the design corresponds in general to the informative dimension, except for some digital tools, such as Open Justice, where Law students offer virtual legal advice, and the GANA Municipales tool, which sometimes provides means of interaction, enabling popular vote for community projects.

Keywords: e-government, social participation, civil society, information technology, Colombia.

\section{Introducción}

El artículo despliega una clasificación propia de los tipos de participación ciudadana que se pretende establecer en el diseño del portal electrónico de la Gobernación de Nariño. En un primer momento se presentan teorías de la democracia y de las TIC que respaldan las clases de participación ciudadana identificadas en el gobierno electrónico: informativa, inclusiva, interactiva y de control. Seguidamente se exponen los resultados de la revisión de normativa, las entrevistas y los grupos focales con actores claves.

La clasificación de la participación ciudadana se fundamenta en teóricos de la democracia, entre ellos Sousa Santos (2004), Prezeworki et al. (1998), Sartori (1999), Levy (1999), Márquez (2001), García (2014), Harto de Vera (2006), Subirats (2002), O’Donnell (2010), Peschard (2017), Heidinger et al. (2010) y Lee et al. (2019) como sus principales.

Los resultados de investigación permiten afirmar que el diseño de participación ciudadana del portal electrónico de la Gobernación de Nariño en general corresponde a la clase de participación informativa, a excepción de herramientas digitales que la integran, como Justicia Abierta y GANA Municipales, que en algún momento se dirigen a la clase de participación interactiva identificada en el presente estudio.

\section{Marco teórico}

Hoy en día los portales electrónicos surgen como una oportunidad para la comunicación entre gobierno y sociedad. El mecanismo incorpora las nuevas tecnologías de la información y la comunicación (TIC) a espacios de administración de gobierno, con el 
propósito de mejorar los procesos de participación. De aquí que relaciones de proximidad entre ciudadanos y gobierno aporten al desarrollo de políticas públicas acordes a las necesidades de la sociedad.

Para contextualizar el concepto de participación ciudadana, Boaventura de Sousa Santos se refiere a «influir directamente en las decisiones y controlar las mismas. Si estamos en una nueva fase en el país, es posible y es preciso que el movimiento comunitario avance e influya directamente, presentando propuestas discutidas y definidas» (Sousa Santos, 2004, p. 34).

La definición de participación directa que formula Sousa Santos (2004) se complejiza en las sociedades actuales por el número de ciudadanos que las componen. Uno de los mecanismos que se utilizan en las democracias para participar, según Sartori, es la representación, que se desarrolla en tres direcciones: «a) con la idea de mandato o de delegación; b) con la idea de representatividad; c) con la idea de responsabilidad» (Sartori, 1999, p. 257).

De esta manera, el gobierno electrónico es creado por los Estados-nación con el fin de mejorar los procesos de representación entre gobernados y gobernantes en torno a la gestión pública. Se sustenta en tres principios básicos de la teoría de la democracia: participación, ciudadanía y representatividad, los cuales se encuentran plasmados de manera implícita en los lineamientos del gobierno electrónico.

A fin de comprender hacia qué tipo de participación se dirige el gobierno electrónico del departamento de Nariño, es pertinente profundizar en su clasificación, que se abstrae de las teorías que fundamentan la participación ciudadana, los principios básicos de las TIC y de gobierno electrónico y gobierno abierto.

Las clases de participación ciudadana que se identifican según sus fundamentos son: participación inclusiva, que busca reducir brechas sociales; participación interactiva, dirigida a mejorar los procesos de gestión pública; participación de control, destinada a fortalecer los canales de representación, y participación informativa, dirigida a difundir datos públicos.

\section{Participación informativa}

La clase de participación informativa, según postulados de García (2014), se refiere a la agilización de trámites desde los gobiernos hacia los ciudadanos como un mecanismo para la rendición de cuentas. Se considera que la transmisión de información por los gobiernos mejora la comunicación con los ciudadanos.

Igualmente, este tipo de participación, según autores como Harto de Vera (2006) y Subirats (2002), facilita información de gobierno a la sociedad con el fin de ampliar el conocimiento de los ciudadanos en procesos de gestión pública y mecanismos de control. 
La participación asociada a la información se sustenta en el principio de transparencia, en vista de que la información puede ser manipulada por agentes de gobierno. De este modo, demanda canales legales que supervisen su calidad, como señala O'Donell (2010), en los que los funcionarios públicos, además de desempeñar sus funciones, fiscalicen su cumplimiento entre todos los ciudadanos.

Por eso es importante que, en procesos de transmisión de información de los gobiernos a los ciudadanos, se alejen influencias e intereses particulares de quienes tienen el poder en los mecanismos de participación electrónica.

\section{Participación inclusiva}

La clase de participación inclusiva apunta al acceso igualitario a los canales de comunicación digital. De esta forma, demanda una reducción de las brechas sociales en el acceso a servicios públicos, canales de interacción y de información que brinda el gobierno.

La participación inclusiva se relaciona directamente con procesos de información simétrica que se quiere alcanzar entre ciudadanos y gobierno, dado que existen asimetrías en la información según el grado de poder que manejen las personas. La participación inclusiva como oposición a la desigualdad busca reducir todo tipo de brechas sociales y económicas que limiten la participación ciudadana (Fountain, 2001).

De las consideraciones anteriores surge la necesidad de promover procesos inclusivos para quienes desconocen el manejo de las nuevas tecnologías de la información, lo que demanda procesos de actualización. Como menciona Levy (1999), es necesario que las personas estén capacitadas para discernir la información de forma crítica. De igual forma, el gobierno electrónico debe acompañarse de herramientas que aporten a la redistribución de recursos para la sociedad.

\section{Participación interactiva}

La participación interactiva se relaciona con la comunicación que debe establecerse entre gobierno y ciudadanos para aportar a la gestión pública de determinada sociedad. Es aquella participación que se focaliza en un espacio socioestatal. Su propósito es reactivar el principio de representatividad a través de dispositivos de participación electrónica. 
A partir de autores como Márquez (2001) y Peschard (2017), se puede decir que la participación interactiva demanda una planificación y una organización de los dispositivos de participación electrónica que consienta el desarrollo de portales electrónicos acordes a las necesidades de la ciudadanía.

La participación interactiva se concibe alrededor de un trabajo colaborativo entre funcionarios del gobierno y la ciudadanía, mediante mecanismos legales que aporten al cumplimiento de procesos de interacción de forma coherente. Se refiere a una clase de participación que va más allá del acceso a la información; demanda una comunicación activa entre distintos actores sociales para la toma de decisiones de Estado-nación.

\section{Participación de control}

Por último, la clase de participación de control apunta a mecanismos de rendición de cuentas que garanticen la participación transparente en el campo político. Según Manin (1998) y Sartori (1999), la rendición de cuentas sirve como un medio de participación que admite controlar y amonestar actividades de afectación pública a partir de la teoría de contrapesos. De ahí la importancia de crear plataformas que canalicen información en doble vía para activar mecanismos de rendición de cuentas.

A su vez, los mecanismos de rendición de cuentas demandan una voluntad democrática que facilite medios de control ciudadano ante el gobierno y solicitan una participación activa de los ciudadanos ante las propuestas gubernamentales, que pueden contener información que haya sufrido algún tipo de manipulación.

La manipulación de información es un tema complejo que dificulta la participación. Heidinger et al. (2010) distinguen al menos dos formas de manipulación: por una parte, los servidores públicos utilizan las redes en nombre de las administraciones, se difunde información acordada, y, por otra parte, los servidores públicos pueden publicar información de interés propio. De este modo, se requiere de un conducto institucional que permita discernir los datos y su constatación.

Si, por un lado, el Estado no hace cumplir los derechos y responsabilidades de la ciudadanía y si, por el otro, los ciudadanos no se organizan para hacer valer sus derechos y obligar al Estado a que observe eficientemente sus responsabilidades, el concepto mismo de ciudadanía carece de sentido. (Przeworski et al.,1998, p. 63)

Desde la misma perspectiva de Prezeworki et al. (1998), Lee et al. (2019) comprenden la importancia de la apropiación de dispositivos de participación que disponen 
los gobiernos. De esta forma, el uso de dispositivos de participación electrónica demanda una gestión transparente y responsable que garantice el uso apropiado de medios de comunicación.

\section{Metodología}

En un primer momento se realizó una revisión de normas que respaldan el diseño de participación ciudadana en portales electrónicos. Seguidamente, para detallar la percepción y las iniciativas de participación ciudadana de los diseñadores del portal electrónico de la Gobernación de Nariño, el estudio se apoyó en la información obtenida de entrevistas a colaboradores y funcionarios del portal y de tres grupos focales que presentan puntos de vista de los usuarios.

Se realizaron 11 entrevistas a actores clave que participaron en el diseño y forman parte de la implementación del portal GANA. Se les formularon 18 preguntas relacionadas con las clases de participación que se identifican en el presente estudio. Se preguntó sobre el concepto de participación, sobre la participación inclusiva y la participación de control, referida a canales de rendición de cuentas.

Posteriormente se indagó sobre medios de comunicación que permiten la interacción entre ciudadanos y funcionarios públicos a través del portal electrónico, referidos al tipo de participación interactiva. Por último, se preguntó sobre las fortalezas y debilidades de la plataforma en cuanto a participación. El propósito de las entrevistas fue comprender si el diseño de la plataforma privilegia una o más clases de participación ciudadana.

En los grupos focales participaron 36 personas, a quienes se les plantearon 10 preguntas. Se conformaron tres grupos de 12 integrantes: un grupo de personas provenientes de municipios rurales de la provincia de Obando de Nariño y un grupo de personas del municipio de Pasto, pertenecientes a distintas comunas, que a su vez se subdividió en un grupo perteneciente a comunas del sector Norte y otro del sector Sur. El propósito fue comprender en qué lugares hubo mayor y menor uso del portal electrónico de la Gobernación de Nariño y establecer el diseño de participación.

\section{Resultados y discusión}

\section{Respaldo legal de gobierno electrónico}

El apartado presenta un análisis de normas del contexto internacional, nacional y local que respaldan e institucionalizan el gobierno electrónico en el departamento de 
Nariño. Los documentos se analizan a partir de la revisión de los datos y servicios que ofrece la plataforma del departamento de las TIC Colombia y el perfil del portal electrónico de la Gobernación de Nariño.

Los lineamientos internacionales que amparan la utilización de las TIC en los aparatos burocráticos se encuentran en la Alianza para el Gobierno Abierto (OGP). En Colombia, el gobierno electrónico se respalda en los lineamientos del departamento de las TIC (Manual de gobierno digital). Por último, en el ámbito local se encuentra la política de Gobierno Abierto de Nariño (GANA), aprobada en diciembre de 2018, que aporta a los fundamentos del gobierno electrónico.

En el ámbito internacional, en 2011 se declaró la OGP, respaldada por más de 70 países que se proponen desarrollar herramientas que promuevan el uso de las TIC en los procesos participativos. «Un ejemplo es la plataforma de participación ciudadana Cónsul, creada con código abierto por el ayuntamiento de Madrid, que ha sido replicada en casi 80 localidades del mundo» (Gobernación de Nariño, 2018, p. 11).

En América Latina se ha tratado de impulsar el uso de tecnologías para procesos participativos en distintos países. Uno de ellos es Argentina, donde se ha creado la Agencia de Innovación y Gobierno Abierto, que busca ampliar la información de gestión pública. Otro caso es Chile, donde se han impulsado instrumentos que fomentan la participación ciudadana, «centrados principalmente en ofrecer servicios a los ciudadanos y herramientas de gobierno en línea» (Gobernación de Nariño 2018, p. 11).

Colombia, por su parte, trabaja el gobierno electrónico desde la perspectiva del gobierno digital, bajo la dirección del Ministerio de Tecnologías de la Información y Comunicaciones. El gobierno electrónico en Colombia se legalizó por el Decreto Único Reglamentario del Sector de las Tecnologías de la Información y Comunicaciones 1078, de 2015.

Los portales electrónicos de gobierno en Colombia se sustentan en cuatro componentes: servicios en línea de alta calidad, impulso del empoderamiento y la colaboración de los ciudadanos en el gobierno, mejoramiento de la gestión pública, garantía de la seguridad y privacidad de información.

Los cuatro componentes de los portales electrónicos tienen como propósito alcanzar los más altos estándares de excelencia mediante el retorno de la confianza de las personas por el uso de servicios en línea. De esta forma, los portales electrónicos, a través del desarrollo y la modernización del aparato burocrático, buscan optimizar los canales de comunicación entre el sector público y los ciudadanos.

Entre los trámites que facilita el gobierno electrónico de Colombia se encuentran la descarga y el apoyo en el diligenciamiento de formularios. «La entidad prioriza los procesos de optimización, automatización de los trámites y servicios con base en la caracterización de sus usuarios» (Manual de gobierno en línea, 2018).

En el ámbito local, el gobierno electrónico se concibe como una parte esencial de la política Gobierno Abierto de Nariño, surgida en 2015 con el programa del gobernador 
Camilo Romero y concretada en el plan de desarrollo «Nariño, corazón del mundo» (2016-2019). La política promueve el acceso a información pública, la participación y la colaboración ciudadana (Gobernación de Nariño, 2018, p. 14).

De esta manera, el gobierno electrónico se respalda en la aprobación de la política pública de Gobierno Abierto de Nariño, aprobada en diciembre de 2018. La política se sustenta en la necesidad de fortalecer los procesos de rendición de cuentas y apertura a la información basada en la trasparencia, la participación y la colaboración mediante el uso de las TIC.

El fácil acceso a la información a través de portales electrónicos se concibe como un aporte a procesos de participación ciudadana, dado que, según García (2014), el conocimiento de la gestión pública desarrolla en las personas sentido de pertenencia respecto a las decisiones que toma el gobierno. Se considera que esta clase de participación informativa mejora los canales de comunicación entre los gobiernos y la ciudadanía.

En sí, la revisión documental en los ámbitos internacional, nacional y específico de Nariño demuestra la prevalencia de la clase de participación informativa en los portales electrónicos de gobierno, mediante herramientas dirigidas a informar a la comunidad y facilitar los trámites.

Según autores como Harto de Vera (2006) y Lee et al. (2019), el acceso a la información es un avance importante del gobierno electrónico, por cuanto permite ampliar el conocimiento de la ciudadanía. No obstante, debe acompañarse de procesos educativos e infraestructura para que las personas absorban la información que brindan los portales electrónicos de una forma crítica y reflexiva, lo que implica que se empoderen por medio del conocimiento.

\section{Entrevistas a funcionarios públicos y colaboradores del portal electrónico de la Gobernación de Nariño}

El desarrollo de las entrevistas señala que el diseño de participación ciudadana del portal electrónico de la Gobernación de Nariño se orienta sobre todo a la clase de participación informativa. Si bien los ideales del portal GANA se orientan a ampliar canales de transparencia, en la práctica la plataforma se convirtió en un medio para albergar gran cantidad de información.

Los diseñadores del portal conciben la participación como un proceso de apropiación de los asuntos de gestión pública por la ciudadanía. Por ejemplo, para el coordinador de Gobierno Abierto, la participación «surge de peticiones formalizadas por colectivos ciudadanos» (Comunicación personal, marzo, 2019).

Los entrevistados comprenden que la participación ciudadana se relaciona con procesos de involucramiento de las personas en asuntos sociales y de gestión pública. 
Manifiestan que esta participación depende de la disposición gubernamental y de la apropiación social de herramientas de acceso a la información.

Respecto a la participación inclusiva, los entrevistados mencionan que en la implementación de gobierno electrónico no se realizaron estudios de conectividad a internet de los hogares del departamento de Nariño, así como no hubo capacitaciones previas a las comunidades. La intención de los diseñadores fue implementar el dispositivo digital y posteriormente mejorar su accesibilidad.

De lo anterior se deduce que el portal electrónico de la Gobernación de Nariño se diseñó para uso general, sin tener en cuenta las diferencias geográficas, de acceso a internet, sociales y culturales. No se llevó a cabo un estudio previo a la creación de herramientas digitales que permitiera conocer las necesidades de las personas.

Como menciona Fountain (2001), el uso de las TIC por los gobiernos tendría que contribuir a la reducción de brechas sociales y económicas. Los dispositivos digitales deberían operar como estrategias para acercar los gobiernos a los ciudadanos mediante canales efectivos de participación que garanticen los derechos humanos y civiles.

En cuanto a las preguntas referidas a los mecanismos para la rendición de cuentas del portal electrónico, que corresponden a la clase de participación de control, puede decirse que no hay herramientas que vayan más allá de brindar información. Tampoco existe un control especializado de los espacios de opinión. Cada dependencia de la Gobernación de Nariño se encarga de responder por un buzón interno.

Para una investigadora del centro de educación superior ESAP y colaboradora del portal, existen herramientas que aportan a procesos de control, como la estrategia Busco Hechos, donde los ciudadanos pueden opinar sobre la información que se publica. Otra herramienta que aporta y que se está ajustando es Justicia Abierta, que brinda asesorías jurídicas gratuitas virtuales. «Las herramientas en mención permiten la vigilancia de las obras por parte de los ciudadanos» (Comunicación personal, marzo 2019).

De este modo, los mecanismos del portal que contribuyen a la participación de control y al proceso de rendición de cuentas consisten sobre todo en la presentación de informes de gestión pública, cifras de presupuesto y asesorías jurídicas que aportan a la transparencia en procesos de gestión.

En referencia a los vacíos que presenta la plataforma en materia de mecanismos de control, se advierte la ausencia de canales de supervisión de procesos de gestión pública. No existen medios que admitan un seguimiento de proyectos y garanticen amonestaciones públicas a través de los espacios virtuales. Para Sartori (1999), los canales de participación deberían incluir canales de comunicación que permitan dar un seguimiento efectivo a los procesos de gestión.

Respecto a la clase de participación interactiva, los diseñadores del portal señalan que hasta el momento no se han desarrollado canales que admitan interacción directa entre funcionarios de gobierno y ciudadanos. Sin embargo, medios que contribuyen en 
esta dirección son el chat institucional y la herramienta Justicia Abierta (consultorios jurídicos).

Para un académico de la Universidad de Nariño y colaborador de la plataforma, la herramienta Justicia Abierta contribuye a procesos de interacción en la medida en que establece espacios de consultas ciudadanas a través de chats básicos. «Nariño es líder en estos proyectos que articulan la gobernación y las universidades. Se presta atención y asesoramiento permanente en temas jurídicos» (Comunicación personal, marzo 2019).

De lo anterior se desprende que, si bien el portal electrónico brinda herramientas de interacción como Justicia Abierta y facilita espacios de opinión en las herramientas digitales, la plataforma aún presenta vacíos en cuanto a canales de comunicación bidireccional dirigidos a reactivar el principio de representatividad.

Autores como Márquez (2001) y Peschard (2017) consideran que la participación interactiva demanda una disposición burocrática de dispositivos electrónicos acordes a las necesidades de la ciudadanía. Deben ser herramientas de fácil acceso que canalicen las solicitudes de los ciudadanos y contribuyan a dar respuestas justificadas.

Por último, se preguntó a los diseñadores del portal sobre la forma de medir la participación ciudadana por este medio. Se los consultó, además, sobre las herramientas digitales que tienen mayor uso y sobre las fortalezas y debilidades del portal electrónico en cuanto a participación. Los entrevistados afirmaron que la participación se mide a través del número de visitas al sitio.

En referencia a las fortalezas de la plataforma GANA, los diseñadores y colaboradores mencionaron que facilita el acceso a la información pública, y la información contribuye a la transparencia de la gestión. Como debilidades señalaron la escasa apropiación de la información por los ciudadanos y la ausencia de indicadores que permitan medir la participación.

De esta manera, entre las ventajas del portal se identificó el fácil acceso a la información de la Gobernación de Nariño. No obstante, se presenta como dificultad la escasa apropiación ciudadana de las herramientas digitales y de la información, lo cual muchas veces se relaciona con la falta de capacitación y educación en el manejo de medios ofimáticos, aunque también puede deberse a la falta de interés de los ciudadanos en los informes.

De allí la importancia de promover la apropiación ciudadana de herramientas digitales que brinda el gobierno. «Es importante entender cómo el intercambio de información entre la administración y los ciudadanos puede transformar significativamente la forma en que opera la esfera pública» (Criado y Rojas-Martín, 2015, p. 48).

\section{Grupos focales usuarios portal electrónico de la Gobernación de Nariño}


Para complementar la información recolectada de las entrevistas a funcionarios públicos del portal electrónico, se conformaron tres grupos focales de 12 usuarios, uno perteneciente a la subregión de Obando y dos al municipio de Pasto, residentes en diferentes comunas, quienes participaron en una jornada de votación presupuestal en la Universidad de Nariño.

El grupo de Obando comprende el concepto de participación ciudadana como el seguimiento de los ciudadanos a los procesos públicos y las políticas de los departamentos.

Respecto al uso del portal electrónico, los participantes consideran escaso su recorrido por la plataforma, dado que «no se ha hecho el intento o no se ha tenido la necesidad de usarla; la única herramienta que se conoce es GANA Municipales, que permite la elección digital de proyectos en las comunidades» (Grupo focal 1, comunicación personal, junio 2019).

Destacan como una fortaleza del portal electrónico, y particularmente de la herramienta GANA Municipales (participación presupuestal a través de dispositivos digitales que son llevados hasta las comunidades), la comunicación bidireccional que permite entre ciudadanos y actores de gobierno para la elección de proyectos.

En cuanto a los inconvenientes, los participantes señalan el escaso acceso a internet, lo cual afecta la comunicación de muchos municipios de Nariño. Es el caso de la subregión de Obando, donde la señal es baja y además limitada. «Únicamente pueden acceder a internet las personas que cancelan un plan» (Grupo focal 1, comunicación personal, junio 2019).

En resumidas cuentas, el grupo focal dirigido a participantes pertenecientes a la subregión de Obando de Nariño resalta la necesidad de seguir aplicando estrategias participativas presenciales como GANA Municipales con el fin de avanzar en procesos democráticos.

El grupo focal de residentes del sector Norte de Pasto comprende la participación ciudadana como un proceso de incidencia política popular cuya mayor expresión es el voto. Por ello manifiesta que herramientas digitales como GANA Municipales son una buena opción para activar la comunicación entre los gobiernos y las comunidades (Grupo focal 2, comunicación personal, junio 2019).

En cuanto al uso del portal electrónico de la Gobernación de Nariño, los participantes lo conocen muy poco. Saben que existe una página de la Gobernación, pero no han utilizado la mayoría de las herramientas digitales. «En su pensar se tiene que los portales electrónicos albergan una cantidad de información que no puede ser discernida» (Grupo focal 2, comunicación personal, junio 2019).

Los participantes rescatan del portal la herramienta GANA Municipales, dado que involucra a la comunidad en la elección de proyectos y es un instrumento accesible para las personas a través de las visitas de los funcionarios a las localidades. 
Los miembros de este grupo consideran que el mayor inconveniente que presenta el portal es la falta de publicidad de las herramientas. «Para el caso de los GANA Municipales, si bien permiten la elección de proyectos por parte de los ciudadanos, un problema que presentan es la esteticidad del proceso» (Grupo focal 2, comunicación personal, junio 2019).

El grupo focal de habitantes del sector Sur del municipio de Pasto entiende la participación ciudadana como un mecanismo para la rendición de cuentas. «La participación ciudadana se desprende del sentido de pertenencia y compromiso social de todas las personas. De esta manera, no pueden decidirse políticas y programas de gobierno sin consultarse primero al pueblo» (Grupo focal 3, comunicación personal, junio 2019).

En referencia al portal electrónico, los participantes mencionan que le han dado muy poco uso a la plataforma, puesto que «la Gobernación no ha realizado difusión de la dirección electrónica al ciudadano» (Grupo focal 3, comunicación personal, junio 2019).

Del portal reconocen la herramienta GANA Municipales, dado que su uso por las comunidades ha sido más factible. Resaltan de la estrategia el uso de dispositivos virtuales que se acercan a la comunidad y dinamizan la elección de proyectos.

Como inconvenientes, los participantes señalan la falta de publicidad y la escasa articulación que tiene la Gobernación con los medios de comunicación. «Algo se difundió por medio de las emisoras de la herramienta digital GANA Municipales, mas no se realizó alguna convocatoria que permitiera una apropiación del resto de herramientas» (Grupo focal 3, comunicación personal, junio 2019).

En este sentido, el grupo focal de Pasto sector Sur manifiesta la necesidad de recuperar espacios públicos virtuales, a través de los cuales las personas puedan acceder a servicios e interactuar con el gobierno. Asimismo, sostienen que la implementación de nuevas tecnologías podría acompañarse de capacitaciones con el fin de empoderar a las personas en su uso.

Lo expuesto permite afirmar que el diseño de participación ciudadana del portal electrónico de la Gobernación de Nariño en general corresponde a la clase de participación informativa, a excepción de dos de sus herramientas digitales, GANA Municipales y Justicia Abierta, que se dirigen en cierto modo a la clase de participación interactiva identificada en el presente estudio.

No obstante, en términos de participación interactiva en portales electrónicos, se requiere una disposición burocrática que aporte a la socialización, la capacitación y la actualización de las personas en medios digitales. Ello implica una ciudadanía diferente a la que se ha conocido en las sociedades agrarias y en las sociedades industriales regidas por los Estados-nación, es decir, requiere transitar de la ciudadanía urbana e industrial, hoy en día dominante, a la infociudadanía (Echeverría, 2001, p. 90). 
El tránsito de la ciudadanía a la infociudadanía demanda el reconocimiento de las redes como una reproducción del espacio público. Para Lee et al. (2019, p. 42), «Los espacios virtuales se han ido creando siguiendo en parte la imagen de los espacios urbanos». Por tanto, es necesario que ciertas pautas del espacio virtual den respuesta a los procesos de representatividad política. La interacción entre gobierno y ciudadanos debería ocupar un espacio central en las nuevas sociedades del conocimiento.

\section{Conclusiones}

En general, las políticas y la normativa de gobierno electrónico en los ámbitos internacional, nacional y en el caso específico de Nariño demuestran que los principios de transparencia, colaboración y participación giran en torno a aspectos informativos. Las herramientas de participación se dirigen sobre todo a facilitar trámites e informar a la comunidad de avances de gestión pública.

Tanto las estrategias como los contenidos que se brindan desde el portal electrónico de la Gobernación de Nariño se orientan en su mayoría a la clase de participación informativa, excepto herramientas como Justicia Abierta y GANA Municipales, que en algún momento se dirigen a la clase de participación interactiva. El acceso a la información es concebido como un mecanismo transparente para la rendición de cuentas.

La herramienta digital Justicia Abierta (consultorios jurídicos) es una estrategia que contribuye a la interacción entre ciudadanos y gobierno. Pese a ello, carece de mecanismos de control de los casos atendidos. Tampoco visibiliza técnicas de encuestas que permitan la retroalimentación de la herramienta.

GANA Municipales es una herramienta que permite la comunicación bidireccional entre funcionarios públicos y ciudadanos en un momento determinado. Asimismo, permite acercar dispositivos digitales a las comunidades más alejadas del departamento de Nariño para la elección de proyectos. Una dificultad que presenta es la falta de seguimiento de la ejecución de proyectos, aspecto en el que la incidencia ciudadana es mínima.

En general, los portales electrónicos dispuestos por los gobiernos para la participación ciudadana han hecho un importante recorrido en términos de ampliar la información pública. A pesar de eso, el estudio arroja la necesidad de trabajar en mecanismos de control, inclusión e interacción social para fortalecer los procesos democráticos.

\section{Anexos}

\section{Anexo 1. Guion de entrevista}

Objetivo: Describir la percepción e iniciativas de participación ciudadana de los diseñadores y colaboradores del portal electrónico de la gobernación de Nariño. 
Datos de entrevistados: Nombre, edad, profesión y municipio en el que habitan.

\section{Concepción de participación ciudadana}

1. ¿Cómo concibe la participación ciudadana?

\section{Medios de participación}

2. ¿Cuál es la cobertura del portal electrónico de la gobernación de Nariño?

3. ¿Se capacitó en medios ofimáticos a los ciudadanos de Nariño desde el portal electrónico de la Gobernación? ¿Cómo?

4. ¿Se capacitó a las personas en uso del portal electrónico? ¿Cómo?

\section{Diseño de participación ciudadana en gobierno electrónico}

5. ¿Cómo se estructura el portal electrónico de la Gobernación de Nariño?

6. La información que se publica ¿ofrece algún contacto para plantear dudas e inquietudes?

7. ¿Cómo funcionan los buzones de solicitudes para resolver dudas e inquietudes?

8. La información que se brinda en el portal ¿tiene algún tipo de supervisión estatal?

9. La información que se brinda en el portal ¿cuenta con canales de transparencia?

10. ¿El portal brinda herramientas para mantenerse en contacto con agentes de gobierno? ¿Cuáles?

11. ¿Qué mecanismos de comunicación tienen los usuarios para interactuar con entidades públicas?

12. ¿El portal brinda mecanismos para la participación en propuestas, programas y políticas públicas?

13. ¿El portal electrónico prevé auditorías virtuales? ¿Cómo se participa?

14. ¿Qué procesos de rendición de cuentas adelanta el portal electrónico de la Gobernación?

15. ¿Cuáles es el uso más relevante que han dado los usuarios al gobierno electrónico?

16. ¿Qué aspectos considera inciden en la participación ciudadana?

17. ¿Cuáles son las fortalezas del portal en materia de participación ciudadana?

18. ¿Cuáles son las debilidades del portal en materia de participación ciudadana?

\section{Anexo 2. Guion para la realización del grupo focal}


Objetivo: Caracterizar los contenidos y las estrategias de participación ciudadana que desarrolla el portal electrónico de la Gobernación de Nariño.

\section{Concepción de participación ciudadana}

1. ¿Cómo conciben la participación ciudadana?

\section{Medios de participación}

2. ¿Con que medios de comunicación cuentan en su hogar (computador, internet...)?

3. ¿Reciben capacitación en manejo de medios ofimáticos? ¿En cuáles?

4. ¿Saben cómo utilizar el portal electrónico de la Gobernación?

\section{Diseño de participación ciudadana en gobierno electrónico}

5. ¿Cómo se estructura el portal electrónico de la Gobernación?

6. La información que se publica ¿ofrece algún contacto para establecer dudas e inquietudes?

7. ¿Los buzones de solicitudes son eficaces para resolver dudas e inquietudes?

8. ¿Usted sabe si la información que brinda el portal tiene algún tipo de supervisión estatal?

9. ¿Considera que la información que se brinda desde el portal electrónico es confiable?

10. ¿El portal brinda herramientas para mantenerse en contacto con agentes de gobierno? ¿Cuáles?

11. ¿Han utilizado algún mecanismo de comunicación con entidades públicas? ¿Cuáles?

12. ¿El portal brinda mecanismos para la participación en propuestas, programas y políticas públicas?

13. ¿Conocen si el portal prevé auditorías virtuales? ¿Cómo se participa?

14. ¿Han participado en algún proceso de rendición de cuentas del portal electrónico? ¿Cómo es el proceso?

\section{Finalmente}

15. ¿Cuáles es el uso más relevante que han dado al portal electrónico?

16. ¿Qué aspectos considera inciden en la participación ciudadana?

17. ¿Cuáles son las fortalezas del portal en materia de participación ciudadana?

18. ¿Cuáles son las debilidades del portal en materia de participación ciudadana? 


\section{Referencias bibliográficas}

Criado, J. I., y Rojas-Martín, F. (2015). Estrategias y realidades en la difusión de las redes sociales en las administraciones públicas: Análisis desde una perspectiva institucionalista. Revista de Ciencias Administrativas y Sociales, 25(57), 45-62.

Echeverría, J. (2001). Teletecnologías: Espacios de interacción y valores. En J. A. López Cerezo, J. L. Luján, y E. M. García Palacios (eds.), Filosofía de la tecnología. Madrid: OEI.

Fountain, J. (2001). The virtual state: Transforming american government? National Civic Review, 90(3), 241-251.

García, J. (2014). Gobierno abierto: Transparencia, participación y colaboración en las Administraciones Públicas. Revista de Ciencias Administrativas y Sociales, 24(54), 75-88.

Gobernación de Nariño. (2018). Borrador de política pública Gobierno Abierto. (No publicado).

Harto de Vera, F. (2006). Tipologías y modelos de democracia. Revista de Internet: Derecho y Política, 2, 32-44.

Heidinger, C., Buchmann, E., y Bohn, K. (2010). Impact assessment in public policy: Towards a Web 2.0 application. Information. Polity: The International Journal of Government \& Democracy in the In-formation Age, 75(1-2), 33-50.

Lee, Y. S., Díaz Puentes, J., y Martin, S. (2019). The contribution of open government to prosperity of society. International Journal of Public Administration, 42(2), 144157. doi: 10.1080/01900692.2017.1405446.

Levy, P. (1999). Qué es lo virtual. Barcelona: Paidós.

Manin, B. (1998). Los principios del gobierno representativo. Madrid: Alianza.

Márquez, P. (2001). La participación ciudadana con relación a la gestión pública. Temas Sociales: Boletín del Programa de Pobreza y Políticas Sociales de SUR, 42. Recuperado de https://www.sitiosur.cl/detalle-de-la-publicacion/?participacion-ciudadana-en-la-gestion-publica-segunda-parte.

Ministerio de Tecnologías de la Información y las Comunicaciones. (2018). Estrategia de gobierno en línea. Recuperado de https://siteal.iiep.unesco.org/sites/default/files/sit_accion_files/co_5005.pdf.

O’Donnell, G. (2010). Democracia, agencia y Estado: Teoría con intención comparativa. Buenos Aires: Prometeo.

Peschard, J. (2017). Transparencia: Promesas y desafíos. México: El Colegio de México.

Przeworski, A., Barfhan, P., Bresser, L. C., Bruszt, L., Choi, J. J., Turkosh Comisso, E. ..., y Wiatr, J. J. (1998). Democracia sustentable. Buenos Aires: Paidós.

Sartori, G. (1999). Elementos de teoría política. Madrid: Alianza.

Sousa Santos, B. de (2004). Democracia de alta intensidad: Apuntes para democratizar la democracia. La Paz: Corte Nacional Electoral. 
Subirats, J. (2002). Los dilemas de una relación inevitable: Innovación democrática y tecnologías de la información y de la comunicación. En H. Cairo Carou (comp.), Democracia digital: Límites y oportunidades (pp. 89-114). Madrid: Trotta. 